\title{
Study of nasal exhaled nitric oxide levels in diagnosis of allergic rhinitis in subjects with and without asthma
}

This article was published in the following Dove Press journal:

Journal of Asthma and Allergy

22 March 2017

Number of times this article has been viewed

\author{
Sy Duong-Quy ${ }^{1-3}$ \\ Thuc Vu-Minh ${ }^{4}$ \\ Thong Hua-Huy' \\ Tram Tang-Thi-Thao ${ }^{3}$ \\ Khiet Le-Quang ${ }^{3}$ \\ Dinh Tran-Thanh ${ }^{3}$ \\ Nhu Doan-Thi-Quynh ${ }^{3}$ \\ Nhat-Nam Le-Dong 5 \\ Timothy J Craig ${ }^{2}$ \\ Anh-Tuan Dinh-Xuan' \\ 'Department of Respiratory \\ Physiology, Cochin Hospital, Paris \\ Descartes University, Sorbonne Paris \\ Cité, Paris, France; ${ }^{2}$ Department \\ of Medicine, Penn State University, \\ Hershey, PA, USA; '3Bio-Medical \\ Research Center, Lam Dong Medical \\ College, Dalat, ${ }^{4}$ Department of \\ Immuno-Allergology, ENT National \\ Institute, Hanoi, Vietnam; ${ }^{5}$ Department \\ of Pulmonology, St Elisabeth Hospital, \\ Namur, Belgium
}

Correspondence: Sy Duong-Quy Bio-Medical Research Center, Lam Dong Medical College, 16 Ngo Quyen, Dalat, Vietnam

$\mathrm{Tel}+84918413813$

$\mathrm{Fax}+84633815000$

Email sduongquy.jfvp@gmail.com
Background: The measure of fractional exhaled nitric oxide (FENO) in the airways is a useful tool to guide the diagnosis and titration of inhaled corticosteroids in patients with asthma. However, its role in diagnosis of allergic rhinitis (AR), especially in subjects with asthma, is not well established.

Objective: To study the cutoff of nasal FENO in the diagnosis of subjects with AR and ARasthma compared to age-matched subjects without AR or asthma and its correlations with the clinical and functional characteristics.

Methods: The study was cross sectional and descriptive. Subjects were grouped into control subjects, AR, and AR-asthma, based on the inclusion criteria. Exhaled NO (nasal FENO, bronchial FENO, and alveolar concentration of NO) was measured by multiple flow electroluminescence device.

Results: Six hundred twenty-eight subjects were included: 217 control subjects (children: $\mathrm{n}=98$, $10 \pm 4$ years; adults: $n=119,50 \pm 16$ years), 168 subjects with AR (children: $n=54,10 \pm 3$ years; adults: $n=114,49 \pm 15$ years), and 243 subjects with AR-asthma (children: $n=115,10 \pm 3$ years; adults: $n=128,51 \pm 14$ years). Nasal peak inspiratory flow and peak expiratory flow were lower in subjects with AR and AR-asthma than in control subjects $(P<0.01$ and $P<0.01$; and $P<0.05$ and $P<0.01$, respectively). Nasal FENO levels were significantly higher in subjects with AR and AR-asthma than in control subjects $(1614 \pm 629$ and $1686 \pm 614$ ppb vs $582 \pm 161 \mathrm{ppb} ; P<0.001$ and $P<0.001$, respectively). In subjects with AR non-asthma, the cutoffs of nasal FENO for those diagnosed with AR were $775 \mathrm{ppb}$ in children, $799 \mathrm{ppb}$ in adults, and 799 in the general population (sensitivity: $92.68 \%, 92.63 \%$, and $92.65 \%$, respectively; specificity: $91.67 \%, 95.00 \%$, and $96.87 \%$, respectively). In subjects with AR-asthma, the cutoffs of nasal FENO were higher, especially in asthma children (1458 ppb; sensitivity: $72.97 \%$ and specificity: $95.83 \%$ ).

Conclusion: Nasal FENO measurement is a useful technique for the diagnosis of AR in subjects with and without asthma.

Keywords: nitric oxide, NO, FENO, nasal FENO, allergic rhinitis, asthma

\section{Introduction}

Allergic rhinitis (AR) is a very common disease that affects approximately $25 \%$ of the world's population and its prevalence continues to increase. ${ }^{1}$ In the US, 20-40 million people are diagnosed with AR and some data suggest approximately $40 \%$ of children have AR. ${ }^{2}$ In Europe, the estimated prevalence of AR is $23 \%$ of the population. ${ }^{3}$ In Vietnam, the prevalence of AR in the general population (GP) has not been reported until now. However, unpublished data presented at Vietnamese Ear-Nose-Throat (ENT) Congress in 2016 showed that the prevalence of AR in local Vietnamese population varied from $14.9 \%$ to $39.7 \%$. The symptoms of AR include 
rhinorrhea, nasal pruritus, sneezing, and nasal congestion. AR may be seasonal, perennial, or intermittent, depending on exposure and sensitization. AR is often associated with allergic symptoms of the eyes (conjunctivitis), skin (eczema), and lower airways (asthma). Most patients with asthma have concurrent AR and up to $40 \%$ of AR patients have asthma. ${ }^{4,5}$ Recent studies showed that the level of asthma control in patients with AR and asthma is correlated with the severity of AR. ${ }^{6,7}$

The high prevalence of AR in patients with asthma is often referred to as the one airway theory since both are characterized by influx of eosinophils, mast cell degranulation, and upregulation of Th2-related cytokine production from the lymphocytes. ${ }^{8}$ For this reason, patients with AR with or without asthma frequently are assessed for airway hyperresponsiveness (challenge with reversibility), spirometry for airway obstruction, skin testing for hypersensitivity to allergens, total and specific IgE, and fractional exhaled nitric oxide (FENO) measurement.

Nitric oxide (NO) is produced mainly by the nasal and bronchial epithelial and inflammatory cells under the control of inducible NO synthase. In subjects with AR, nasal FENO is triggered principally by airborne allergens (pollens, house dust mite, insects, or pets). ${ }^{9,10}$ Similar to the lower airway, the concentration of nasal FENO can be measured by constant aspiration or expiratory flows by nasal mask. ${ }^{11-13}$ Studies demonstrated that in healthy subjects and in subjects with AR, the values of nasal FENO are dependent on aspiration and expiratory flow rate. However, in subjects with AR, especially who have associated asthma, the diagnosed values of nasal FENO are not well defined.

This study was planned to determine the cutoffs of nasal FENO in Vietnamese subjects with AR or AR-asthma, compared to healthy controls, and the correlation between nasal FENO and the clinical and functional characteristics in these patients.

\section{Subjects and methods Subjects}

Subjects more than 5 years old living in Vietnam, who came to the Clinical Research Center of Lam Dong Medical College for diagnosis and treatment of AR or for asthma and met the inclusion and exclusion criteria, were included in this study after they signed an Institutional Review Board approved consent. For subjects under 18 years, the parents or guardians signed the informed consent. This study has been approved by Lam Dong Medical College Institutional Review Board.

\section{Exclusion criteria}

Study subjects having one of the following criteria were excluded from the study: acute or chronic cardiorespiratory diseases (acute myocardial infarction, severe coronary disease, chronic heart failure, chronic obstructive pulmonary disease), severe asthma exacerbations needing systemic corticosteroid therapy at screening, AR or asthma treated currently with nasal or inhaled corticosteroids, septal deviation or nasal polyp diagnosed by ENT doctors, and subjects unable to perform functional tests or measurement of FENO.

\section{Inclusion criteria}

AR: the clinical diagnosis of AR (made by ENT doctors) was made when patients had one or more of the symptoms (nasal congestion, nose running, nasal itching, or sneezing) lasting more than 4 days per week and occurring when exposed to allergens (dog or cat allergen, pollen, fungus, house dust mites) in the environment or workplace (exposure to certain chemicals, irritants, or allergens at work). ${ }^{14}$

Asthma: the clinical diagnosis of asthma was based on the Global Initiative for Asthma guidelines with a history of respiratory symptoms such as wheeze, shortness of breath, chest tightness, and cough that varies over time and in intensity. ${ }^{15}$ The spirometry confirmed the reversibility of airway obstruction.

Control: age-matched healthy subjects were included in this cross-sectional and control study after they or their parents or guardians (for subjects under 18 years old) signed an approved consent. Healthy subjects were excluded from the present study if they had one of the following criteria: other allergic diseases, symptoms of AR or asthma, or positive skin prick test.

\section{Methods}

\section{Study design}

This study was a cross-sectional and descriptive study. The study subjects were classified as control, AR, and AR-asthma groups according to the inclusion and exclusion criteria. All data on medical history, family history, clinical examination, skin tests, lung function, and exhaled nitric oxide parameters were collected for statistical analyses.

\section{Laboratory techniques}

Skin prick test (Stallergenes, London, UK): negative control was $0.9 \%$ saline solution and positive control was $1 \mathrm{mg} / \mathrm{mL}$ of histamine. Six respiratory allergens including Dermatophagoides pteronyssinus (Dp), Dermatophagoides farinae (Df), Blomia tropicalis (Blo), dog dander, cat 
dander, cockroach, Phoenix dactylifera, Alternaria spp, and mixed pollens (Dactylis glomerata, Phleum pratense, Lolium perenne) were used for testing. Percutaneous skin test was considered positive when the wheal size exceeded the negative control by $3 \mathrm{~mm}$.

Lung function testing was done using Koko Spirometer (nSpire Health, Inc., Longmont, CO, USA) or with Body Box 500 (Medisoft, Sorinnes, Belgium) for whole-body phlethysmography. The reversibility of forced expiratory volume in 1 second $\left(\mathrm{FEV}_{1}\right)$ was evaluated 15 min posttreatment with $200 \mu \mathrm{g}$ (for children) or $400 \mu \mathrm{g}$ (for adult) salbutamol. The reversal was positive when there was an increase of FEV1 $\geq 12 \%$ and $200 \mathrm{~mL}$.

Nasal peak inspiratory flow (PIF) and nasal peak expiratory flow (PEF) were measured with Mediflux Device (Mediflux, Paris, France).

\section{Exhaled NO}

Nasal exhaled NO (nasal FENO) was measured with constant aspiratory flow using the Hypair FeNO ${ }^{+}$Device (Medisoft), which is an electrochemical based analyzer. The procedure is as follows: 1) patient connects a catheter to one of his/her nostrils; 2) air from the nasal cavity is continuously analyzed by the NO electrochemical sensor; and 3) throughout the analysis, patient breathes through an expiratory brake, so that the velum is closed to prevent any contamination of nasal air with bronchial air. The aspiration flow rate was $100 \mathrm{~mL} /$ second through a nasal catheter.

For the airways, bronchial FENO and alveolar concentration of $\mathrm{NO}(\mathrm{CANO})$ were measured at multiple flow rates (50, 100,150 , and $350 \mathrm{~mL} /$ second). Technical measurement of exhaled NO was taken according to manufacturer's instructions, as recommended by the American Thoracic Society/ European Respiratory Society guideline and the results of exhaled NO had been reported by Hypair FeNO+ Expair Software (Medisoft). ${ }^{15}$

\section{Statistical analyses}

SPSS 22.0 software (IBM Corporation, Armonk, NY, USA) was used to analyze these data. Categorical variables were expressed as numbers or percentages. Continuous variables were presented as mean \pm standard deviation (SD). Normal distribution was tested using skewness-kurtosis. Regression analysis was used to measure the correlation between nasal FENO and continuous variables, with the correlation coefficient $R$ of Pearson for normal distribution variables (PIF, PEF, bronchial FENO, and CANO) and of Spearman for non-normal distribution variables (age, height, weight, body mass index
[BMI], AR symptoms, and lung function parameters). The pair wise comparison of mean was performed using MannWhitney $U$ test, and comparison of more than two groups was done with Kruskal-Wallis test. One-way analysis of variance was used to determine whether there were any statistically significant differences between the mean values of three or more independent (unrelated) groups. Youden index with area under the curve (AUC) was used to determine the cutoff of nasal FENO for diagnosis of AR in patients with or without asthma. MedCalc software (Ostend, Belgium) with DeLong method was used to compare the AUC between groups.

\section{Results}

\section{Clinical and functional characteristics of the study subjects}

From January 2015 to May 2016, 628 subjects were included in the present study, including 217 control subjects, 168 subjects with AR (AR non-asthma), and 243 subjects with AR-asthma. The anthropometric and clinical and functional characteristics of the study subjects are presented in Table 1. There were no significant differences between the three groups in mean age, male to female ratio, or BMI. The AR symptoms were significantly different between subjects with AR vs those with AR-asthma, with predominance of nasal congestion in the AR-asthma cohort ( $92 \%$ vs $75 \%, P<0.05$; Table 1 ). There was no significant difference in the percentage of positive skin tests between subjects with AR and those with AR-asthma (Table 1). The FEV 1 was significantly lower in subjects with AR-asthma than in control subjects and subjects with AR nonasthma $(77 \% \pm 23 \%$ vs $97 \% \pm 11 \%$ and $93 \% \pm 12 \%, P<0.001$ and $P<0.01$, respectively; Table 1). Nasal PIF and PEF were significantly lower in subjects with $\mathrm{AR}$ and $\mathrm{AR}$-asthma than in control subjects $(86 \pm 22$ and $89 \pm 37 \mathrm{~L} / \mathrm{min}$ vs $134 \pm 33 \mathrm{~L} / \mathrm{min}$, $P<0.01$ and $P<0.01$; and $134 \pm 50$ and $113 \pm 33 \mathrm{~L} / \mathrm{min}$ vs $175 \pm 48$ L/min, $P<0.05$ and $P<0.01$, respectively; Table 1$)$. The levels of nasal FENO were significantly higher in subjects with AR and AR-asthma than in control subjects (1614 \pm 629 and $1686 \pm 614$ ppb vs $582 \pm 161 \mathrm{ppb} ; P<0.001$ and $P<0.001$, respectively).

\section{Correlation between nasal FENO, clinical and functional characteristics, bronchial FENO, and CANO}

There were no significant correlations between nasal FENO and anthropometric characteristics (age, height, weight, and BMI; Table 2). However, there were significant correlations between nasal FENO and clinical symptoms in AR and ARasthma cohorts (Table 2). While the symptoms of sneezing 
Table I Clinical and functional characteristics of study subjects

\begin{tabular}{|c|c|c|c|c|}
\hline Parameters & Control $(n=217)$ & $A R(n=168)$ & AR-asthma $(n=243)$ & $P$-value ${ }^{\mathrm{a}, \mathrm{b}}$ \\
\hline \multicolumn{5}{|l|}{ Clinical characteristics } \\
\hline Age, years & $37 \pm 23$ & $36 \pm 22$ & $31 \pm 20$ & $0.124,^{\mathrm{c}} 0.097,^{\mathrm{d}} 0.266^{\mathrm{e}}$ \\
\hline Male/female ratio & I.I & 1.2 & 0.9 & $0.096,{ }^{c} 0.107,{ }^{d} 0.234^{e}$ \\
\hline $\mathrm{BMI}, \mathrm{kg} / \mathrm{m}^{2}$ & $2 I \pm 4$ & $19 \pm 6$ & $20 \pm 4$ & $0.112,{ }^{c} 0.227,{ }^{d} 0.077^{e}$ \\
\hline AR symptom, ${ }^{f} \%$ & 0 & 100 & 100 & \\
\hline Nasal congestion, $\%$ & - & 75 & 92 & $0.028^{c}$ \\
\hline Rhinorrhea, \% & - & 96 & 78 & $0.022^{c}$ \\
\hline Sneezing and/or nasal itching, \% & - & 93 & 74 & $0.019^{c}$ \\
\hline Asthma symptoms, ${ }^{\circ} \%$ & - & - & 100 & - \\
\hline Positive skin test, ${ }^{h} \%$ & - & 86 & 88 & $0.324^{c}$ \\
\hline \multicolumn{5}{|l|}{ Functional characteristics } \\
\hline FVC, \% & $98 \pm 12$ & $95 \pm 13$ & $82 \pm 22$ & $0.034,^{\mathrm{c}} 0.013,^{\mathrm{d}} 0.082^{\mathrm{c}}$ \\
\hline $\mathrm{FEV}_{1}, \%$ & $97 \pm 11$ & $93 \pm 12$ & $77 \pm 23$ & $0.003,,^{c} 0.0005,^{d} 0.076^{e}$ \\
\hline $\mathrm{FEV}_{1} / \mathrm{FVC}, \%$ & $88 \pm 12$ & $87 \pm 12$ & $86 \pm 17$ & $0.236,^{\mathrm{c}} 0.114,^{\mathrm{d}} 0.238^{\mathrm{e}}$ \\
\hline Nasal PIF, L/min & $134 \pm 33$ & $89 \pm 37$ & $86 \pm 22$ & $0.330,^{c} 0.006,^{d} 0.008^{e}$ \\
\hline Nasal PEF, L/min & $175 \pm 48$ & $134 \pm 50$ & $113 \pm 33$ & $0.089,^{c} 0.007,{ }^{d} 0.035^{e}$ \\
\hline \multicolumn{5}{|l|}{ Exhaled NO } \\
\hline Nasal FENO, ppb & $582 \pm 161$ & $1614 \pm 629$ & $1686 \pm 6 \mid 4$ & $0.087,,^{c} 0.0005,,^{d} 0.0006^{e}$ \\
\hline Bronchial FENO, ppb & $10 \pm 4$ & $12 \pm 6$ & $35 \pm 28$ & $0.0006,,^{c} 0.0003,{ }^{d} 0.066^{e}$ \\
\hline CANO, ppb & $3 \pm 1$ & $3 \pm 2$ & $6 \pm 4$ & $0.015,^{c} 0.017,^{d} 0.088^{c}$ \\
\hline
\end{tabular}

Notes: aKruskal-Wallis test for pair wise comparison of three groups; ${ }^{b}$ Mann-Whitney $U$ test for pair wise comparison of two groups; 'AR-asthma vs AR; ${ }^{\mathrm{d} A R}$-asthma vs control; eAR vs control; thaving at least one symptom (nasal congestion, rhinorrhea, sneezing, and/or nasal itching); gas defined by GINA $2015^{15}$; hskin prick test positive with at least one allergen.

Abbreviations: AR, allergic rhinitis; BMI, body mass index; CANO, concentration of alveolar nitric oxide; FENO, fractional exhaled nitric oxide; FEV , forced expiratory volume in I second; FVC, forced vital capacity; GINA, Global Initiative for Asthma; NO, nitric oxide; PEF, peak expiratory flow; PIF, peak inspiratory flow; Ppb, parts per billion.

Table 2 Correlation between nasal FENO, clinical and functional characteristics, and bronchial FENO and CANO

\begin{tabular}{|c|c|c|c|c|c|c|}
\hline \multicolumn{7}{|l|}{ Nasal FENO (ppb) } \\
\hline \multirow[t]{2}{*}{ Groups } & \multicolumn{2}{|c|}{ Control $(n=217)$} & \multicolumn{2}{|c|}{ AR $(n=168)$} & \multicolumn{2}{|c|}{ AR-asthma $(n=243)$} \\
\hline & $\boldsymbol{R}$ & $P$-value & $\boldsymbol{R}$ & P-value & $\boldsymbol{R}$ & $P$-value \\
\hline \multicolumn{7}{|c|}{ Anthropometric characteristics ${ }^{a}$} \\
\hline Age, years & 0.133 & 0.341 & 0.124 & 0.241 & 0.145 & 0.343 \\
\hline Height, cm & 0.026 & 0.854 & 0.054 & 0.621 & 0.078 & 0.564 \\
\hline Weight, kg & 0.100 & 0.477 & 0.133 & 0.429 & 0.115 & 0.311 \\
\hline $\mathrm{BMI}, \mathrm{kg} / \mathrm{m}^{2}$ & 0.126 & 0.369 & 0.178 & 0.405 & 0.166 & 0.524 \\
\hline \multicolumn{7}{|l|}{ AR symptoms ${ }^{b}$} \\
\hline Nasal congestion & - & - & 0.484 & 0.0014 & 0.786 & 0.0034 \\
\hline Rhinorrhea & - & - & 0.652 & 0.0018 & 0.692 & 0.0023 \\
\hline Sneezing and/or nasal itching & - & - & 0.742 & 0.0029 & 0.629 & 0.0029 \\
\hline \multicolumn{7}{|l|}{ Functional characteristics ${ }^{\mathrm{a}}$} \\
\hline FVC, \% & 0.054 & 0.465 & 0.104 & 0.421 & 0.087 & 0.096 \\
\hline $\mathrm{FEV}_{1}, \%$ & 0.026 & 0.302 & 0.068 & 0.523 & 0.102 & 0.545 \\
\hline $\mathrm{FEV}_{1} / \mathrm{FVC}, \%$ & 0.065 & 0.544 & 0.072 & 0.506 & 0.092 & 0.439 \\
\hline \multicolumn{7}{|l|}{ Exhaled airway $\mathrm{NO}^{\mathrm{a}}$} \\
\hline Bronchial FENO, \% & 0.104 & 0.374 & 0.098 & 0.084 & 0.162 & 0.085 \\
\hline CANO, ppb & 0.098 & 0.132 & 0.045 & 0.089 & 0.103 & 0.168 \\
\hline
\end{tabular}

Notes: aLinear correlation; 'Spearman correlation.

Abbreviations: AR, allergic rhinitis; BMI, body mass index; CANO, concentration of alveolar nitric oxide; FENO, fractional exhaled nitric oxide; FEV , forced expiratory volume in I second; FVC, forced vital capacity; NO, nitric oxide; ppb, parts per billion.

and/or nasal itching were strongly correlated with nasal FENO in subjects with $\operatorname{AR}(R=0.742, P=0.0029$; Table 2$)$, the symptom of nasal congestion was strongly correlated with nasal FENO in subjects with AR-asthma $(R=0.786, P=0.0034$; Table $2)$. There were moderate negative correlations between nasal
FENO, PIF, and PEF in subjects with AR and AR-asthma $(R=-0.482, P=0.001$ and $R=-0.467, P=0.005 ; R=-0.356$, $P=0.002$ and $R=-0.492, P=0.012$, respectively; Figure 1$)$. However, there were no significant correlations between nasal FENO, bronchial FENO, and CANO (Table 2). 

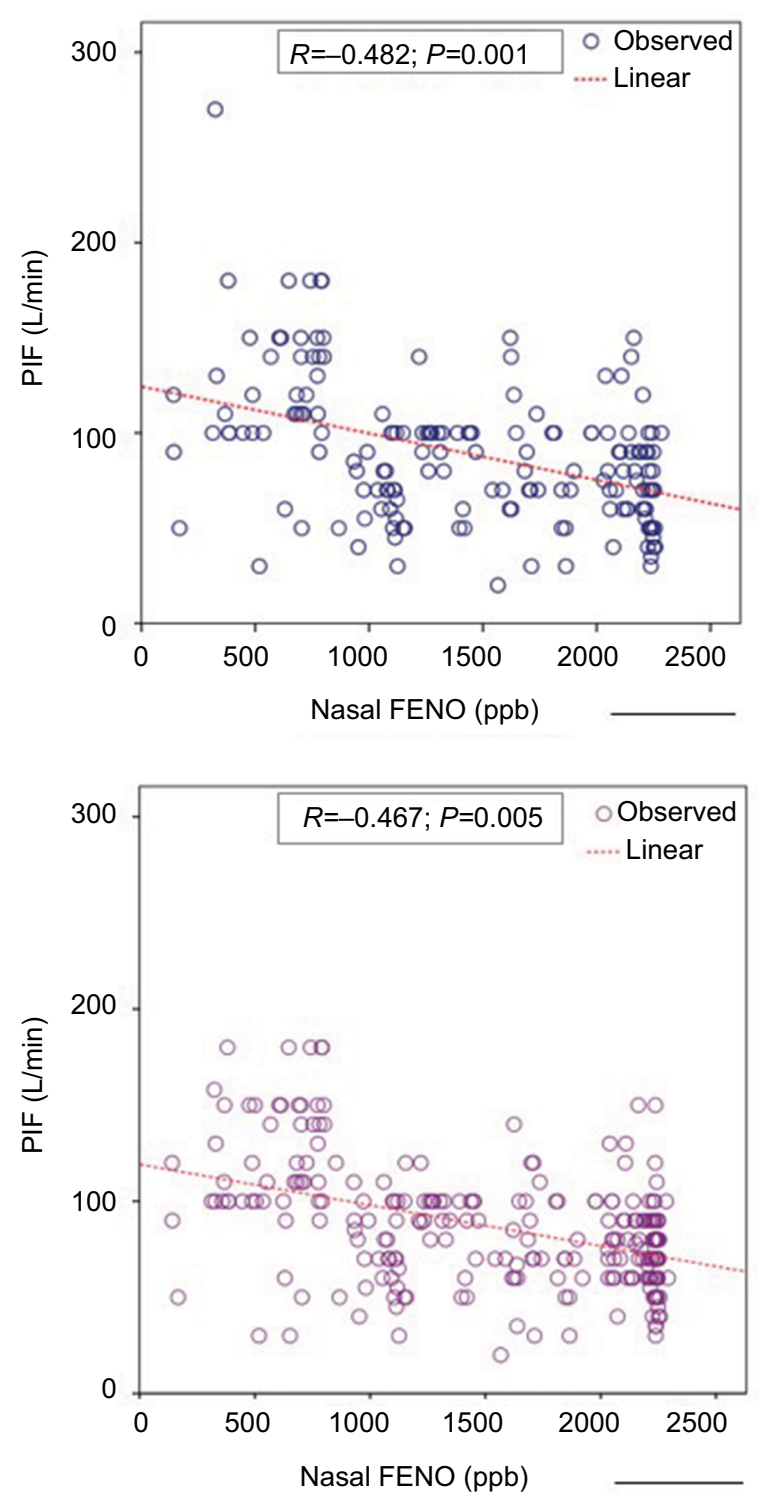
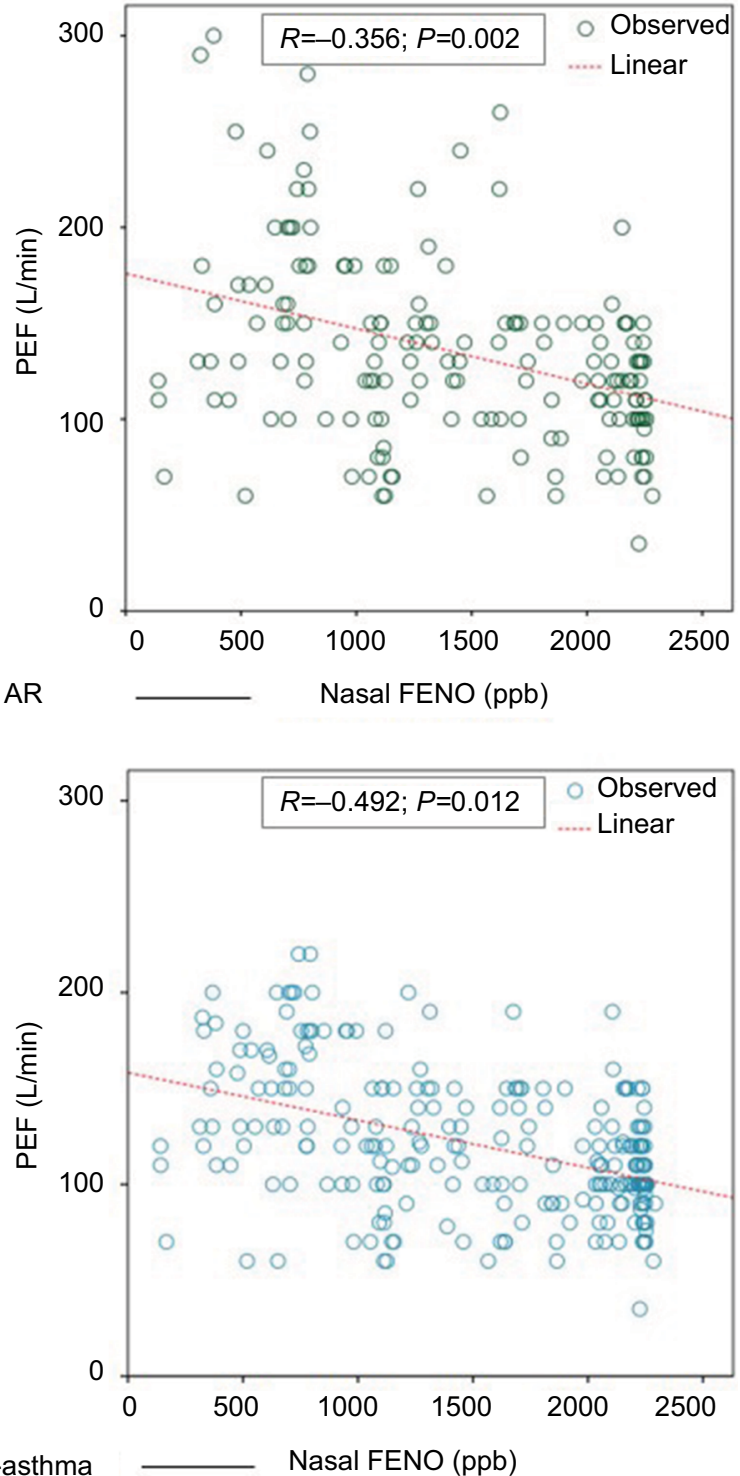

Figure I Correlation between nasal FENO and PIF in subjects with AR (top) and AR-asthma (bottom). Abbreviations: AR, allergic rhinitis; FENO, fractional exhaled nitric oxide; NO, nitric oxide; PEF, peak expiratory flow; PIF, peak inspiratory flow; PPb, parts per billion.

\section{Cutoff of nasal FENO for diagnosis of AR classified by population}

The results of the present study showed that in subjects with $\mathrm{AR}$, the cutoffs of nasal FENO with the highest Youden index were $775 \mathrm{ppb}$ in children, $799 \mathrm{ppb}$ in adults, and $799 \mathrm{ppb}$ for the GP, with high sensitivity $(92.68 \%, 92.63 \%$, and $92.65 \%$, respectively) and specificity $(91.67 \%, 95.00 \%$, and $96.87 \%$, respectively; Table 3). However, in subjects with AR-asthma, the cutoffs of nasal FENO for diagnosis of AR were higher than those in subjects with AR non-asthma, especially in asthmatic children (1458 ppb) with lower sensitivity (72.97\%) and high specificity (95.83\%; Table 3 ).

\section{Comparison of diagnostic values of AUC of nasal FENO}

The AUC values of diagnostic values of nasal FENO were not significantly different between children with AR and with AR-asthma ( $0.947 \pm 0.0317$ vs $0.957 \pm 0.0256, P=0.8072$; Figure 2), adults with AR and with AR-asthma ( $0.956 \pm 0.0195$ vs $0.992 \pm 0086, P=0.0922$; Figure 2$)$, and GP $(0.953 \pm 0.0167$ vs $0.956 \pm 0.0154, P=0.9024$; Table 4 ).

\section{Discussion}

The results of our study showed that: 1) the levels of nasal FENO in subjects with AR and AR-asthma were significantly 
Table 3 Cutoff of nasal FENO for diagnosis of AR classified by population

\begin{tabular}{llllllllll}
\hline Nasal FENO & Cutoff, (ppb) & Sensitivity (\%) & $\mathbf{9 5 \%} \mathbf{C l}$ & Specificity (\%) & $\mathbf{9 5 \%} \mathbf{C l}$ & $\mathbf{+ L R}$ & $\mathbf{9 5 \%} \mathbf{C l}$ & $\mathbf{- L R}$ & $\mathbf{9 5 \%} \mathbf{C l}$ \\
\hline AR & & & & & & & & & \\
Children & 775 & 92.68 & $80.1-98.5$ & 91.67 & $61.5-99.8$ & 11.12 & $1.7-72.8$ & 0.080 & $0.03-0.2$ \\
Adults & 799 & 92.63 & $85.4-97.0$ & 95.00 & $75.1-99.9$ & 18.53 & $2.7-125.3$ & 0.078 & $0.04-0.2$ \\
GP & 799 & 92.65 & $86.9-96.4$ & 96.87 & $83.8-99.9$ & 29.65 & $4.3-204.2$ & 0.076 & $0.04-0.1$ \\
AR-asthma & & & & & & & & & \\
Children & 1458 & 72.97 & $61.4-82.6$ & 95.83 & $78.9-99.9$ & 17.51 & $2.6-119.9$ & 0.28 & $0.2-0.4$ \\
Adults & 990 & 98.47 & $94.6-99.8$ & 92.86 & $66.1-99.8$ & 13.79 & $2.1-91.1$ & 0.016 & $0.004-0.07$ \\
GP & I27I & 82.44 & $76.5-87.4$ & 94.74 & $82.3-99.4$ & 15.66 & $4.1-60.4$ & 0.19 & $0.1-0.3$ \\
\hline
\end{tabular}

Abbreviations: AR, allergic rhinitis; $\mathrm{Cl}$, confidence interval; FENO, fractional exhaled nitric oxide; GP, general population; LR, likelihood ratios; ppb, parts per billion.
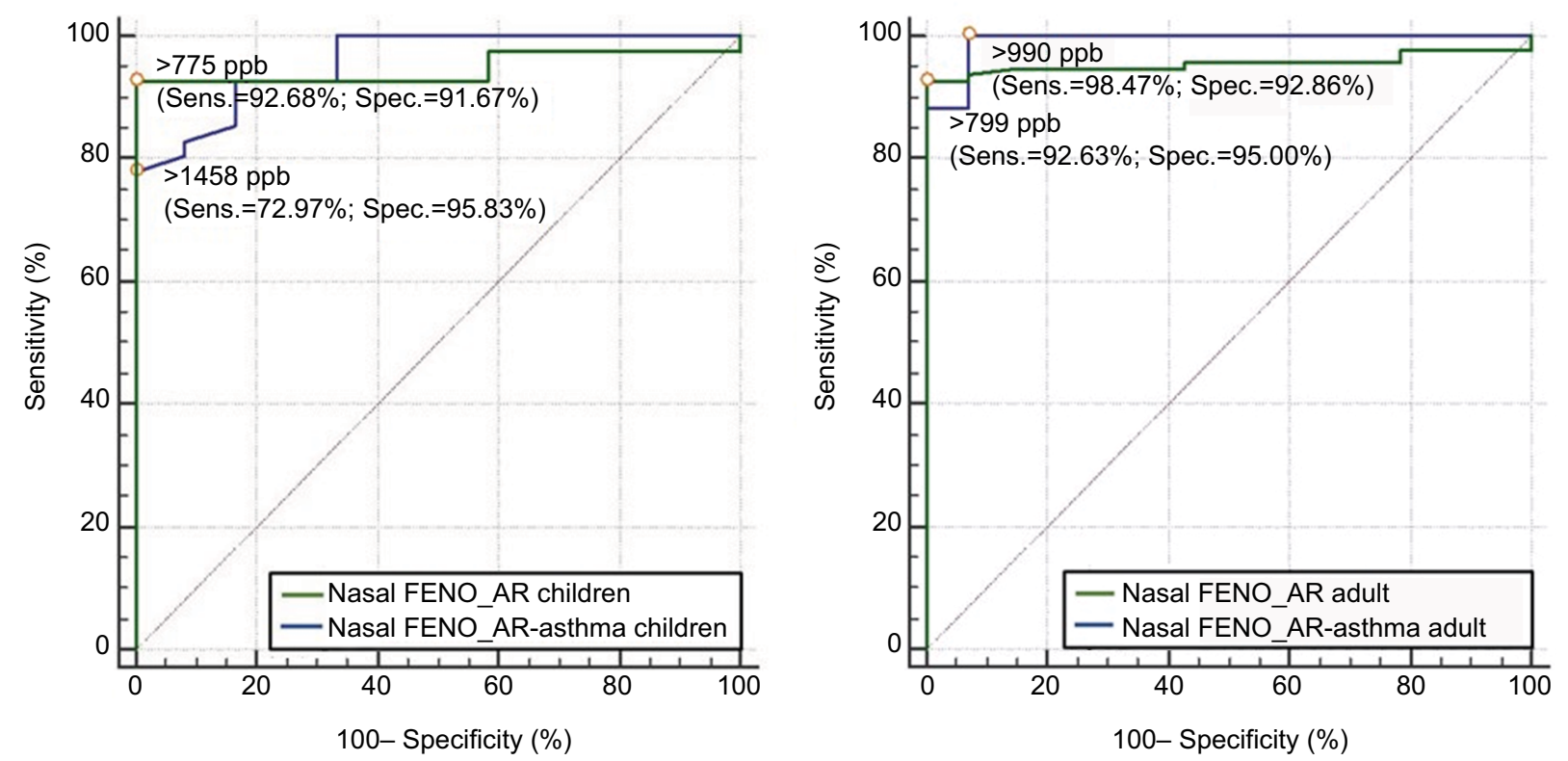

Figure 2 Comparison of AUC of nasal FENO cutoff in children (left) and adults (right) with AR vs AR-asthma.

Note: AUC comparison with DeLong method.

Abbreviations: AUC, area under the curve; AR, allergic rhinitis; FENO, fractional exhaled nitric oxide; Sens., sensitivity; Spec., specificity.

Table 4 Comparison of diagnostic values of nasal FENO between subjects with AR and AR-asthma

\begin{tabular}{|c|c|c|c|c|c|c|c|c|}
\hline Comparison & AUC & SE & $95 \% \mathrm{Cl}$ & Difference of AUC & SE & $95 \% \mathrm{Cl}$ & $Z$ score & $P$-value \\
\hline \multicolumn{9}{|l|}{ Children } \\
\hline$A R$ & 0.947 & 0.0317 & $0.849-0.990$ & 0.0102 & 0.0416 & $-0.0715-0.0918$ & 0.244 & 0.8072 \\
\hline AR-asthma & 0.957 & 0.0256 & $0.863-0.994$ & & & & & \\
\hline \multicolumn{9}{|l|}{ Adults } \\
\hline$A R$ & 0.956 & 0.0195 & $0.899-0.986$ & 0.0357 & 0.0212 & $-0.00586-0.0773$ & 1.684 & 0.0922 \\
\hline AR-asthma & 0.992 & 0.00860 & $0.951-1.000$ & & & & & \\
\hline \multicolumn{9}{|l|}{ GP } \\
\hline$A R$ & 0.953 & 0.0167 & $0.909-0.980$ & 0.00287 & 0.0234 & $-0.0430-0.0488$ & 0.123 & 0.9024 \\
\hline AR-asthma & 0.956 & 0.0154 & $0.913-0.982$ & & & & & \\
\hline
\end{tabular}

Note: AUC comparison with DeLong method.

Abbreviations: AR, allergic rhinitis; AUC, area under the curve; $\mathrm{Cl}$, confidence interval; FENO, fractional exhaled nitric oxide; GP, general population; SE, standard error.

higher than in control subjects; 2) the levels of nasal FENO were significantly correlated with AR symptoms and PIF and PEF; 3) the cutoffs of nasal FENO for diagnosis of ARasthma were higher than in subjects without asthma; and 4) the sensitivity and specificity of the nasal FENO cutoffs for AR diagnosis were not significantly different.
In healthy subjects, the concentration of nasal FENO is higher than that in the lower airways as measured by aspiration (350-750 vs 5-25 ppb). Previous studies demonstrated that in the upper airways, exhaled nasal NO was produced mainly from paranasal sinus. ${ }^{16}$ However, a recent study demonstrated that the nasal exhaled NO was obtained not 
only from the sinuses by gradient diffusion, but also from the NO produced by nasal mucosal membranes. ${ }^{17,18}$ In this study, the levels of nasal FENO in healthy subjects varied from 350 to $750 \mathrm{ppb}$ and were not significantly correlated with anthropometric characteristics, lung functional parameters, or levels of exhaled NO in lower airways (bronchial FENO and CANO; Table 2). This result is very interesting in regard to the use of nasal FENO as an independent parameter for the diagnosis of rhinosinusitis. The use of nasal FENO in diagnosis of AR has been reported in previous studies. ${ }^{13,17}$ Similar to other allergic inflammatory airway diseases such as asthma, in AR, the increase in nasal FENO appears to be related to the upregulation of inducible NO synthase, mainly in epithelial cells and eosinophils. ${ }^{19-22}$

Although the role of nasal FENO in AR has been demonstrated, its role in diagnosis of AR associated with asthma has not been clearly demonstrated. Previous studies using different techniques (electrochemical and chemiluminescence analyzers) have demonstrated elevated NO in the lower airways in obstructive sleep apnea and asthma. ${ }^{23-25}$ The present study showed using electrochemical-based analyzer that the levels of nasal FENO were significantly higher in subjects with AR and AR-asthma than in control subjects, but they were not significantly different between AR and AR-asthma subjects (Table 1). In subjects with AR with or without asthma, the levels of nasal FENO were positively correlated in a linear fashion with AR symptom and negatively correlated in a linear fashion with nasal peak flow rates (Table 2; Figure 1). These data suggest that nasal FENO in combination with clinical symptoms might be a useful tool for diagnosis of AR. Nesic et al also showed that the levels of nasal FENO in AR patients were significantly higher than in healthy individuals and that nasal FENO measurement had an excellent reliability. ${ }^{13}$ Interestingly, the results of our study showed that in subjects with AR-asthma, bronchial FENO and CANO were significantly higher than in healthy subjects and subjects with AR; however, there were no significant correlations with nasal FENO. This suggests that nasal FENO might be used as an objective and independent parameter for diagnosis of $\mathrm{AR}$ in patients with or without other respiratory comorbidities such as asthma. ${ }^{26,27}$ Despite this, the cutoff of nasal FENO for diagnosis of AR in these conditions might be different.

The present study showed that in AR subjects with asthma, these cutoffs were higher for diagnosis of AR, especially in children with AR-asthma (1458 ppb; Table 3; Figure 2). This suggests that the levels of nasal FENO used in diagnosis of AR could be changed in subjects with coexisting inflammatory airway disease such as asthma. The higher level of nasal FENO in subjects with AR associated with asthma might be due to the increased production of $\mathrm{NO}$ from the nasal membrane by the inflammatory status rather than the contamination from lower airway or digestive tract. In the present study, the contamination of NO produced from the mouth and digestive tract was prevented by the mouth breathing counter-pressure system that had been integrated in the NO measurement device. This system helps to close the soft palate of patients during the measure of nasal FENO by aspiration. Inversely, in asthma patients with AR, Chen et al demonstrated that the level of bronchial FENO was significantly higher in those with nasal obstruction and the authors suggested that it was due to the relationship between upper and lower airway inflammation rather than a dynamic factor. ${ }^{28}$

However, the results of our study showed that the diagnostic values (sensitivity and specificity) of nasal FENO for AR in subjects with and without asthma were not significantly different (Table 4). This statement was confirmed by comparing the AUC of nasal FENO cutoffs in children and adults with AR vs AR-asthma (Table 4; Figure 2). In asthma patients associated with AR, Guo et al showed that the diagnostic accuracy of FENO for asthma was 0.88 by measuring the AUC by the receiver operating characteristic curves, and the diagnostic odds ratio of AR was only 2.99 (95\% confidence interval, 0.85-10.45). ${ }^{29}$

As the aim of the present study was mainly to determine the cutoff of nasal FENO for the diagnosis AR in asthma subjects in comparison to AR subjects without asthma and controls, we did not study the correlation between the level of nasal FENO and the control of asthma. Another limitation is due to the cross-sectional and descriptive nature of the study, we could not demonstrate the role of nasal FENO in the treatment of AR and in the control of asthma. The preliminary result suggests that the measure of nasal FENO might be useful in clinical practice to diagnose subjects with AR. However, more studies in this field are necessary in the future to clarify the role of nasal FENO in the management of AR.

\section{Conclusion}

The measure of nasal exhaled NO is a feasible and useful method for the diagnosis of AR in healthy subjects and subjects with asthma. The cutoffs of nasal FENO for the diagnosis of AR are different in subjects with asthma from those of subjects without asthma. Hence, it is necessary to perform more studies in a large number of subjects to clarify the role of nasal exhaled $\mathrm{NO}$ in the diagnosis of $\mathrm{AR}$ in the GP and in subjects with asthma. 


\section{Acknowledgment}

The authors would like to thank all the colleagues at the Clinical Research Unit of Lam Dong Medical College for their contribution to this study.

\section{Disclosure}

The authors report no conflicts of interest in this work.

\section{References}

1. Santos CB, Pratt EL, Hanks C, McCann J, Craig TJ. Allergic rhinitis and its effect on sleep, fatigue, and daytime somnolence. Ann Allergy Asthma Immunol. 2006;97(5):579-587.

2. Bhattacharyya N. Incremental healthcare utilization and expenditures for allergic rhinitis in the United States. Laryngoscope. 2011; 121(9):1830-1833.

3. Canonica GW, Bousquet J, Mullol J, Scadding GK, Virchow JC. A survey of the burden of allergic rhinitis in Europe. Allergy. 2007;62(Suppl 85): $17-25$.

4. Bousquet J, Schunemann HJ, Samolinski B, et al. Allergic rhinitis and its impact on asthma (ARIA): achievements in 10 years and future needs. J Allergy Clin Immunol. 2012;130(5):1049-1062.

5. Cruz AA, Popov T, Pawankar R, et al; ARIA Initiative Scientific Committee. Common characteristics of upper and lower airways in rhinitis and asthma: ARIA update, in collaboration with GA(2)LEN. Allergy. 2007; 62(Suppl 84):1-41.

6. Oka A, Matsunaga K, Kamei T, et al. Ongoing allergic rhinitis impairs asthma control by enhancing the lower airway inflammation. J Allergy Clin Immunol Pract. 2014;2(2):172-178.

7. Oka A, Hirano T, Yamaji Y, et al. Determinants of incomplete asthma control in patients with allergic rhinitis and asthma. J Allergy Clin Immunol Pract. 2016. pii: S2213-2198(16)30377-4.

8. Agache I, Akdis CA. Endotypes of allergic diseases and asthma: an important step in building blocks for the future of precision medicine. Allergol Int. 2016;65(3):243-252.

9. Kang BH, Chen SS, Jou LS, Weng PK, Wang HW. Immunolocalization of inducible nitric oxide synthase and 3-nitrotyrosine in the nasal mucosa of patients with rhinitis. Eur Arch Otorhinolaryngol. 2000;257(5):242-246.

10. Yuksel H, Kirmaz C, Yilmaz O, et al. Nasal mucosal expression of nitric oxide synthases in patients with allergic rhinitis and its relation to asthma. Ann Allergy Asthma Immunol. 2008;100(1):12-16.

11. Liu D, Huang Z, Huang Y, Yi X, Chen X. Measurement of nasal and fractional exhaled nitric oxide in children with upper airway inflammatory disease: preliminary results. Int J Pediatr Otorhinolaryngol. 2015;79(12):2308-2311.

12. Takeno S, Okabayashi Y, Kohno T, Yumii K, Hirakawa K. The role of nasal fractional exhaled nitric oxide as an objective parameter independent of nasal airflow resistance in the diagnosis of allergic rhinitis. Auris Nasus Larynx. 2016. pii: S0385-8146(16)30369-8.

13. Nesic VS, Djordjevic VZ, Tomic-Spiric V, Dudvarski ZR, Soldatovic IA, Arsovic NA. Measuring nasal nitric oxide in allergic rhinitis patients. J Laryngol Otol. 2016;130(11):1064-1071.
14. Seidman MD, Gurgel RK, Lin SY, et al. Clinical practice guideline: allergic rhinitis executive summary. Otolaryngol Head Neck Surg. 2015; 152(2):197-206.

15. Global Initiative for Asthma (GINA); 2015. Available from: http:// ginasthma.org/archived-reports/. Accessed April 11, 2016.

16. Lundberg JO, Farkas-Szallasi T, Weitzberg E, et al. High nitric oxide production in human paranasal sinuses. Nat Med. 1995;1(4): 370-373.

17. Scadding G, Scadding GK. Update on the use of nitric oxide as a noninvasive measure of airways inflammation. Rhinology. 2009;47(2): 115-120.

18. Cho WS, Kim TH, Kim KH, et al. Increased expression of arginase I and II in allergic nasal mucosa. Laryngoscope. 2011;121(2): 236-240.

19. Bautista AP, Eisenlohr CP, Lanz MJ. Nasal nitric oxide and nasal eosinophils decrease with levocetirizine in subjects with perennial allergic rhinitis. Am J Rhinol Allergy. 2011;25(6):383-387.

20. Irander K, Palm JP, Borres MP, Ghafouri B. Clara cell protein in nasal lavage fluid and nasal nitric oxide - biomarkers with anti-inflammatory properties in allergic rhinitis. Clin Mol Allergy. 2012;10:4.

21. American Thoracic Society; European Respiratory Society. ATS/ ERS recommendations for standardized procedures for the online and offline measurement of exhaled lower respiratory nitric oxide and nasal nitric oxide, 2005. Am J Respir Crit Care Med. 2005;171(8): 912-930.

22. Dinh-Xuan AT, Annesi-Maesano I, Berger P, et al; French Speaking Respiratory Society. Contribution of exhaled nitric oxide measurement in airway inflammation assessment in asthma. A position paper from the French Speaking Respiratory Society. Rev Mal Respir. 2015;32(2):193-215.

23. Duong-Quy S, Hua-Huy T, Tran-Mai-Thi HT, Le-Dong NN, Craig TJ, Dinh-Xuan AT. Study of exhaled nitric oxide in subjects with suspected obstructive sleep apnea: a pilot study in Vietnam. Pulm Med. 2016; 2016:3050918.

24. Nguyen-Thi-Bich H, Duong-Thi-Ly H, Thom VT, et al. Study of the correlations between fractional exhaled nitric oxide in exhaled breath and atopic status, blood eosinophils, FCER2 mutation, and asthma control in Vietnamese children. J Asthma Allergy. 2016;9: $163-170$.

25. Hua-Huy T, Le-Dong NN, Duong-Quy S, Luchon L, Rouhani S, Dinh-Xuan AT. Increased alveolar nitric oxide concentration is related to nocturnal oxygen desaturation in obstructive sleep apnoea. Nitric Oxide. 2015;45:27-34.

26. de Groot EP, Nijkamp A, Duiverman EJ, Brand PL. Allergic rhinitis is associated with poor asthma control in children with asthma. Thorax. 2012;67(7):582-587.

27. Krantz C, Janson C, Borres MP, Nordvall L, Alving K, Malinovschi A. Nasal nitric oxide is associated with exhaled $\mathrm{NO}$, bronchial responsiveness and poor asthma control. J Breath Res. 2014;8(2):026002.

28. Chen IC, Lin YT, Hsu JH, Liu YC, Wu JR, Dai ZK. Nasal airflow measured by rhinomanometry correlates with FeNO in children with asthma. PLoS One. 2016;11(10):e0165440.

29. Guo Z, Wang Y, Xing G, Wang X. Diagnostic accuracy of fractional exhaled nitric oxide in asthma: a systematic review and meta-analysis of prospective studies. J Asthma. 2016;53(4):404-412.
Journal of Asthma and Allergy

\section{Publish your work in this journal}

The Journal of Asthma and Allergy is an international, peer-reviewed open access journal publishing original research, reports, editorials and commentaries on the following topics: Asthma; Pulmonary physiology; Asthma related clinical health; Clinical immunology and the immunological basis of disease; Pharmacological interventions and

\section{Dovepress}

new therapies. This journal is included in PubMed. The manuscript management system is completely online and includes a very quick and fair peer-review system, which is all easy to use. Visit http://www. dovepress.com/testimonials.php to read real quotes from published authors. 